\title{
Prix de vente des fermes au Québec. Divergence de vue entre les besoins des cédants et des repreneurs
}

Farm Assets Pricing in Quebec: The Discrepancy Between the Needs of the Retiring Farmer and the Successor

David Ouellet et Jean-Philippe Perrier

\section{(2) OpenEdition}

Journals

Édition électronique

URL : http://journals.openedition.org/economierurale/5412

DOI : 10.4000/economierurale. 5412

ISSN : 2105-2581

Éditeur

Société Française d'Économie Rurale (SFER)

Édition imprimée

Date de publication : 31 mars 2018

Pagination : 65-80

ISSN : 0013-0559

Référence électronique

David Ouellet et Jean-Philippe Perrier, «Prix de vente des fermes au Québec. Divergence de vue entre les besoins des cédants et des repreneurs », Économie rurale [En ligne], 363 | janvier-mars 2018, mis en ligne le 31 mars 2020, consulté le 05 janvier 2020. URL : http://journals.openedition.org/ economierurale/5412 ; DOI : 10.4000/economierurale.5412

(c) Tous droits réservés 


\title{
Prix de vente des fermes au Québec Divergence de vue entre les besoins des cédants et des repreneurs
}

\author{
David OUELLET • Jean-Philippe PERRIER • Université Laval, Département d'économie \\ agroalimentaire et sciences de la consommation, Traget Laval, Québec, Canada \\ david.ouellet.6@ulaval.ca ; jeanphilippe.perrier@eac.ulaval.ca
}

L'écart croissant entre les valeurs marchande et économique des exploitations agricoles québécoises complexifie le processus de transfert des fermes à la prochaine génération. Cette étude applique une approche d'évaluation d'entreprises agricoles propre au contexte de leur transmission en vue d'analyser la détermination du prix de vente au transfert. Les résultats montrent que le prix de vente est significativement corrélé aux besoins de retraite nets des cédants et, de façon moins significative, à la valeur économique des capitaux propres des entreprises de l'échantillon. D'importants compromis sont réalisés en vue d'assurer le transfert et, en dépit de leur plus forte capitalisation, les fermes laitières semblent confrontées aux mêmes défis que les autres types de production.

MOTS-CLÉS : transfert de fermes, planification financière, prix de vente d'entreprises, évaluation $d^{\prime}$ entreprises agricoles, entreprises agricoles du Québec

\section{Farm Assets Pricing in Quebec: The Discrepancy Between the Needs of the Retiring Farmer and the Successor}

The growing gap between the market and economic values of Quebec farms makes the process of transferring farm assets to the next generation ever more complex. The present study applies a farm business evaluation method tailored to farm succession in order to analyze the selling price determination. Results show that the selling price in the sample is significantly correlated to the seller's net retirement needs and to a less significant degree, to the economic value of farm equity. Considerable compromises are made on both sides of the transaction in order to insure the transfer and in spite of greater capitalization, dairy farms seem to be faced with similar challenges as other types of production. (JEL: Q12, Q14)

KEYWORDS: farm succession, financial planning, farm assets pricing, farm business valuation, Quebec agricultural businesses

D epuis une trentaine d'années, nous constatons une augmentation marquée de la valeur des actifs agricoles au Québec, plus particulièrement celle des terres. Outre l'effet de la hausse des prix des denrées et de la rareté, la spéculation et l'engouement pour le foncier agricole ont contribué à gonfler la valeur de cet actif (Meloche et Debailleul, 2013). La hausse de la valeur des terres se traduit également par une hausse de la valeur des garanties que représentent les actifs fonciers déjà possédés par les agriculteurs (Meloche et

Debailleul, 2013) et permet donc, dans une certaine mesure, d'augmenter l'acquisition d'actifs via l'endettement, renforçant les pressions inflationnistes sur les prix des autres actifs. Cette tendance contribue à creuser l'écart entre la valeur marchande des actifs composant l'entreprise agricole et leur capacité à produire des bénéfices (Montel et Sanscartier, 2013; Perrier, 2013).

Étant donné cet écart croissant, la ferme peut difficilement se vendre au prix reflétant la pleine valeur marchande des actifs 
qui la composent puisqu'un tel investissement ne pourrait être rentabilisé. Afin d'assurer le transfert de son entreprise à la prochaine génération, l'agriculteur-cédant se voit souvent dans l'obligation d'accepter un prix de vente en deçà de sa valeur marchande (Handfield et al., 2008). Le don d'une partie de la valeur de la ferme constitue donc un élément prépondérant au montage financier lors du transfert (Cantin et Lepage, 2006). Au Québec, le rachat de la ferme par la relève se finance principalement par emprunt (Perrier et al., 2004 ; Tondreau et al., 2002). Cependant, les conditions financières des exploitations se détériorent depuis plusieurs années (Perrier, 2013), ce qui limite la capacité d'emprunt des repreneurs. Certains cédants envisagent alors de financer euxmêmes une partie de la transaction par l'octroi de prêts à conditions favorables. Cette stratégie d'aide au transfert est toutefois contrainte par leurs propres besoins financiers à la retraite. En effet, la propension à l'épargne des agriculteurs étant plutôt faible, la vente d'actifs agricoles représente pour plusieurs un important moyen de financement de la retraite (Montel et Sanscartier, 2013). Par ailleurs, l'augmentation de la valeur des actifs permet à l'agriculteur approchant la retraite d'obtenir un rendement sur gain en capital souvent supérieur à celui obtenu sur un investissement visant une augmentation des revenus récurrents (Hargreaves et McCarthy, 2010). Considérer l'entreprise comme un « fonds de pension » apparaît dès lors antithétique à sa «reprenabilité ». De plus, Pitts et al. (2009) rapportent que, par souci d'équité envers la fratrie, les enfants non-repreneurs sont souvent compensés ${ }^{1}$

1. Contrairement aux cas d'autres pays, notamment celui de la France, lors d'une succession, la loi canadienne ne définit pas les règles de partage de l'héritage entre les héritiers, sauf en l'absence d'un testament légalement valide. La compensation est donc volontaire. dans le partage du patrimoine à la suite du transfert de la ferme. Cette compensation peut engendrer des pressions supplémentaires sur les ressources dont disposent les propriétaires, compensation qui, dans le cas de la ferme moyenne, apparaît comme une obligation incompatible à sa viabilité (Barclay et al., 2007). La conciliation entre les besoins des cédants, la capacité financière des repreneurs, l'équité envers les enfants non-repreneurs et la pérennité de l'entreprise compliquent la dynamique de transfert et peuvent être la source de conflits non négligeables. Déterminer un prix de vente de sorte à ne léser personne peut effectivement s'avérer un exercice fort complexe.

Cet article se base sur des travaux réalisés grâce à une aide financière du Programme Innov'Action agroalimentaire, un programme issu de l'accord Cultivons l'avenir 2, conclu entre le ministère de l'Agriculture, des Pêcheries et de l'Alimentation du Québec (MAPAQ), et Agriculture et Agroalimentaire Canada. Les travaux visaient à analyser la fixation du prix de vente au transfert de fermes du Québec issues de tous les types de production. Une comparaison entre le cas particulier des fermes laitières et celui des autres secteurs a été effectuée, compte tenu du poids de la production laitière dans l'agriculture québécoise et de l'importance de la valeur des quotas au bilan de ce type d'exploitation ${ }^{2}$. L'étude visait également à vérifier empiriquement la conceptualisation du prix de vente proposée par Perrier et al. (2015) où le prix est principalement fonction des besoins de retraite des cé-

2. Euvrant sous le système de gestion de l'offre, les exploitations laitières du Québec doivent détenir des droits de production à hauteur d'environ 17000 euros par kg de matière grasse produite par jour. Un troupeau de taille moyenne ( 60 vaches en lactation) nécessiterait un investissement d'environ un million d'euros pour l'achat de quota seulement. 
dants et de la capacité de remboursement de l'entreprise. Une approche d'évaluation d'entreprises agricoles propre au contexte de transmission à la prochaine génération et s'enquérant des deux côtés (cédants et repreneurs) de transactions véritables, c'est-à-dire ayant été réellement effectuées, a donc été développée. Le présent article se décline de la manière suivante. Une brève revue de la littérature traitant du don consenti et de la détermination du prix de vente d'exploitations agricoles lors du transfert est proposée. La modélisation élaborée dans le cadre de cette étude est ensuite détaillée et la méthodologie d'enquête est présentée. Enfin, la modélisation est vérifiée empiriquement, les résultats sont décrits et discutés.

\section{Transfert et don des exploitations agricoles au Québec}

Le transfert de propriété représente un stade critique dans le développement de l'entreprise (Barclay et al., 2007 ; Gaté et Latruffe, 2016; Lobley, 2010). Payer un prix trop élevé figure d'ailleurs parmi les nombreux facteurs d'insuccès (Parent et al., 2000). S'intéressant à la problématique de transfert et de démantèlement des exploitations agricoles québécoises, Perrier et al. (2004) ont réalisé une étude exploratoire utilisant la méthode de comparaison multicas, étant donné le peu de documentation sur le sujet à l'époque. Les auteurs constataient alors que les entreprises de leur échantillon se vendaient entre 16 et $69 \%$ de la valeur marchande des capitaux propres au transfert, pour une moyenne de $50 \%$. Les difficultés financières rencontrées par ces exploitations étant vraisemblablement la cause du don consenti (Perrier et al., 2004). Plus tard, Handfield et al. (2008) ont analysé les déterminants du don au repreneur lors $\mathrm{du}$ transfert d'exploitations agricoles au Québec. Sondant les intentions des agriculteurs quant à une transaction éventuelle (donc hypothétique), les résultats de l'étude ont montré que la moitié des répondants prévoyait de donner entre 26 et $50 \%$ de la ferme, tandis que seulement $5,3 \%$ prévoyaient de vendre leur ferme à la pleine valeur marchande lors du transfert. L'étude s'ajoutait à celle de Handfield (2006) qui analysait les déterminants du don, suggérant que les cédants conservent une certaine marge de manœuvre quant à l'ampleur du don consenti. La promesse d'une gestion responsable et compétente de la ferme par le repreneur, ainsi que la gratuité, la quantité et la qualité de son travail pendant les années précédant son établissement constitueraient autant de facteurs pouvant exercer une influence sur la détermination du prix de vente, et incidemment sur l'ampleur du don à consentir (Handfield, 2006). Plus récemment, Lepage et al. (2015) se sont penchés sur le cas des fermes laitières du Québec afin d'analyser la capacité de l'exploitation à s'endetter pour réaliser un transfert (rachat des capitaux propres). L'étude montrait que seulement $1 \%$ des entreprises de leur échantillon pouvaient être rachetées à leur pleine valeur marchande tandis que $85 \%$ des fermes ne pouvaient être rachetées à plus de $50 \%$ de leur valeur marchande. Constatant l'écart de plus en plus important entre valeurs marchandes et bénéfices engendrés par la ferme laitière moyenne, les auteurs suggéraient que d'ici 2020, plusieurs fermes laitières n'auront plus la capacité financière d'être rachetées à l'interne et ne seront donc plus «reprenables ».

Considérant les contraintes financières auxquelles ils font face, il convient de s'interroger quant à la réelle marge de manœuvre dont disposent les cédants quant à l'ampleur du don à consentir. Une perception trompeuse de la «réelle» valeur de leurs entreprises pourrait être à l'origine des difficultés rencontrées lors du processus de transfert et plus particulièrement de la détermination du prix de vente. 


\section{Détermination du prix de vente des fermes lors du transfert}

La fixation d'un prix de vente possible pour une entreprise passe d'abord par une estimation de sa valeur marchande. La valeur marchande se définit généralement comme étant le prix le plus élevé qui peut être obtenu pour les actifs d'une entreprise sur un marché libre entre vendeurs et acheteurs informés, consentants et sans liens privilégiés. Afin d'estimer la valeur marchande, trois approches principales sont généralement utilisées. L'approche par l'actif, ou approche patrimoniale (Barthélémy, 1997), consiste en une sommation de la valeur comptable des différents actifs composant l'entreprise (cheptel, bâtiments, matériel, etc.). Cette approche est la plus simple et les calculs ne requièrent que le bilan de l'entreprise. La valeur comptable des actifs est moins volatile et plus conservatrice (Damodaran, 2001) puisqu'elle se base sur la valeur d'acquisition mais ne considère pas la valeur de l'entreprise comme fonction du contexte dans lequel elle évolue. En effet, les investissements qui ont apporté les actifs de l'entreprise ont été réalisés dans le passé, ce qui fait que leur productivité et leur intérêt pour l'entreprise peuvent avoir changé depuis (Barthélémy, 1997). Afin de refléter l'aspect dynamique de la valeur d'une entreprise, une approche d'évaluation qui se concentre sur les résultats bénéficiaires est nécessaire. L'approche par revenus, ou approche économique (Barthélémy, 1997), consiste en une addition des flux de trésorerie futurs générés par l'actif, actualisés selon un taux d'escompte traduisant le coût d'opportunité lié à l'emploi de cet actif. Cette approche peut prendre différentes formes (Ma et Swinton, 2012) et est souvent utilisée comme méthode secondaire servant à valider les résultats obtenus par l'usage d'une méthode alternative. L'approche par revenus permet entre autres de déterminer l'impact des carences des pratiques de gestion (Eves, 2007), mais demeure relativement complexe et chronophage. Les hypothèses concernant la croissance et les revenus futurs de l'entreprise amènent beaucoup d'incertitude et c'est la raison qui pousse les analystes à opter pour la troisième approche soit celle par multiples (Feldman, 2005). L'approche par multiples peut combiner les approches patrimoniale et économique et consiste à exprimer la valeur d'une entreprise (ou d'un actif) sous la forme d'un ratio, de sorte à obtenir un nombre pouvant servir de point de comparaison entre entreprises similaires (valeur/hectare, valeur/hectolitre de lait produit, etc.). L'usage de ratios peut cependant mener à des résultats trop élevés si les entreprises comparables sont surévaluées. La subjectivité du choix de l'échantillon constitue donc un des principaux écueils de cette méthode. La technologie utilisée, la structure de coûts ou la fertilité des sols peuvent entraîner des biais importants dans l'estimation de la valeur d'une entreprise via cette méthode.

En théorie, la valeur doit être indépendante de la méthode utilisée pour l'estimer. Cependant, en pratique, tel n'est pas toujours le cas. Surestimer systématiquement la valeur des actifs, par un usage malavisé des méthodes d'évaluation, et les acquérir à fort prix par l'endettement peut effectivement engendrer des problèmes sévères, et ce particulièrement au moment où les conditions d'emprunt commencent à se resserrer (Mishkin, 2008). En conséquence, une augmentation de la valeur marchande des actifs qui ne serait pas accompagnée d'une augmentation proportionnelle ou supérieure des revenus qu'ils produisent occasionnera une diminution de la productivité du capital et une perte d'efficacité due aux coûts de financement accrus pour l'acquisition de ces actifs. À terme, la capacité de remboursement de l'entreprise pourrait ne pas permettre le plein autofinancement du renouvellement 
des immobilisations amortissables ainsi qu'une partie des nouveaux investissements et de ce fait mettre en péril la situation financière de l'exploitation. En l'absence d'une marge de sécurité financière adéquate, l'entreprise agricole ne peut alors faire face aux imprévus et aux fluctuations du marché, compromettant dès lors les chances de survie des exploitations après leur transmission (Lepage et al., 2011).

La pratique d'évaluation dans le domaine agricole s'est traditionnellement appuyée sur l'approche patrimoniale de sommation des valeurs d'actifs. Selon Barthélémy (1997), cette pratique pourrait avoir masqué temporairement les conséquences de l'écart croissant entre la valeur marchande des exploitations et leur capacité à générer des bénéfices. Si la ferme est considérée comme un fonds de pension pour plusieurs, l'évaluer selon l'approche patrimoniale ou l'approche par multiples et négliger l'approche économique pourrait mener à une perception trop optimiste de la situation et en conséquence à un prix de vente trop élevé, limitant ainsi les perspectives d'un transfert réussi.

Par ailleurs, bien que les méthodes d'évaluation d'entreprise puissent servir de guide dans la fixation du prix de vente, des aspects autres qu'économiques ou financiers sont également à considérer dans l'estimation de valeur. Murray (1969) observe la valeur comme étant « la qualité d'un bien quant à sa capacité de satisfaire un besoin humain ». En ce sens, la valeur d'une entreprise agricole est le reflet de sa finalité, de l'ensemble des objectifs que ses propriétaires visent à atteindre. L'agriculture représentant pour plusieurs un mode de vie autant sinon plus qu'une source de revenus, le principal défi quant à l'évaluation d'une entreprise agricole est de quantifier des aspects qui, somme toute, sont non quantifiables ou du moins très subjectifs (Jacques-Jouvenot et Schepens, 2007). Pour Debruyne (2010), « la valeur d'une entreprise est conditionnée par sa capacité à générer des résultats bénéficiaires. » L'auteur ajoute cependant que l'entreprise agricole, visant davantage la pérennisation que la maximisation des profits, devrait pouvoir s'évaluer dans un contexte de généralisation des problèmes de transmission (Debruyne, 2010). Dans un tel contexte, c'est lors du transfert intergénérationnel que devrait s'exprimer la « vraie» valeur d'une entreprise agricole. Cédants et repreneurs ayant chacun leurs propres attentes, intérêts, perceptions et incitatifs, se questionner quant à la valeur de l'entreprise revient à définir un espace de négociation qui tienne compte de ces différents aspects (Barthélémy, 1997).

Du point de vue du cédant, le fait d'avoir choisi le transfert plutôt que le démantèlement reflète un souci quant à la pérennité de l'entreprise (Perrier et al., 2004). Étant donné l'écart croissant entre la valeur marchande et la capacité d'engendrer des bénéfices, cette préférence implique une volonté de vendre à un prix plus faible que celui obtenu dans le cas d'un démantèlement, prix qui se serait alors davantage rapproché de la valeur marchande. Toutefois, le cédant s'orientant vers une nouvelle étape de sa vie fera probablement face à des besoins financiers plus ou moins élevés, dépendamment de ses projets d'avenir et de l'épargne déjà à sa disposition. Ainsi, l'entreprise pourrait avoir aux yeux du cédant ce que Barthélémy (1997) décrit comme la valeur de remploi, c'est-à-dire un montant d'argent destiné à un nouvel usage et procédant directement de la vente de l'entreprise. Le prix de vente pourrait donc être fixé principalement en fonction des besoins de retraite des cédants, incluant une compensation pour les enfants non repreneurs. À cet effet, sachant que la valeur marchande constitue le montant le plus élevé dont il pourrait éventuellement bénéficier pour sa retraite, le cédant pourrait volontairement surévaluer ses dépenses futures de consommation, ou maintenir un niveau d'épargne relativement faible. 
Les besoins de retraite nets du cédant et la valeur marchande seraient dès lors corrélés et constitueraient deux causes communes expliquant le prix de vente. Toutefois, si le prix de vente est réellement corrélé avec les besoins de retraite nets, en maintenant la valeur marchande de la ferme constante, un test de corrélation partielle devrait révéler un coefficient de corrélation positif et significatif.

H1: Le prix de vente est corrélé avec les besoins de retraite nets des cédants

Par ailleurs, le montant minimal que pourraient espérer recevoir les cédants dépend de ce que peut payer le repreneur. Un montage financier typique comporte une certaine combinaison entre les principaux modes d'acquisition soient le don, l'emprunt, les subventions, le financement par le vendeur et l'autofinancement. A priori, différents montages financiers pourraient assurer la viabilité et la rentabilité de l'entreprise à la suite du transfert, dépendamment du contexte organisationnel et financier avec lequel repreneurs et cédants sont aux prises (Cantin et Lepage, 2006). Dans le cas où le repreneur serait dépourvu de moyens financiers significatifs, le montant maximal à payer serait déterminé par ce que Barthélémy (1997) décrit comme la valeur de remboursement, c'est-à-dire le montant d'argent pouvant être emprunté et incidemment remboursé par les revenus mêmes de l'entreprise. Dès lors, une valeur basée sur la capacité de remboursement de l'entreprise et qui tient compte de son endettement actuel (la valeur économique des capitaux propres) apparaît comme étant un critère prépondérant dans la décision du prix de vente du fait de son influence sur la pérennité de l'entreprise. Si le prix est effectivement déterminé en fonction de la capacité de remboursement de l'entreprise, un test de corrélation partielle devrait révéler un coefficient de corrélation positif et significatif entre le prix de vente et la valeur économique des capitaux propres de l'entreprise, en contrôlant sa valeur marchande.

H2 : Le prix de vente est corrélé avec la valeur économique des capitaux propres de l'entreprise

La modélisation du prix de vente (figure 1) élaborée dans le présent article complète donc celle présentée par Lepage et al. (2015) et opérationnalise celle proposée par Perrier et al. (2015) en posant le prix de vente comme étant d'abord fonction des besoins de retraite nets des cédants, et implicitement d'un souci d'équité envers les enfants non-repreneurs. Le prix est également posé comme étant fonction de la capacité de remboursement de l'entreprise, dans le but d'en favoriser la pérennité.

Figure 1. Modélisation du prix de vente

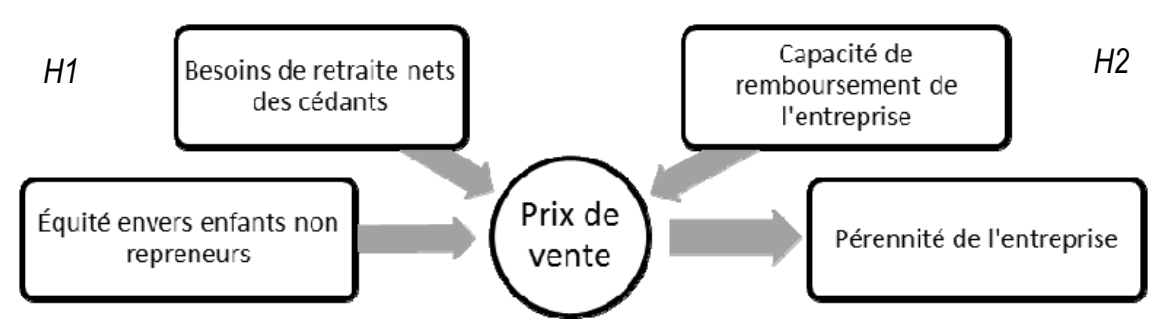

Source : les auteurs. 


\section{Opérationnalisation des variables du modèle et aspects méthodologiques}

Afin de vérifier empiriquement la modélisation et les hypothèses de recherche, des tests de corrélations partielles ont été effectués, chacun validant l'hypothèse posée pour l'un des deux côtés de la transaction. Ce test a été choisi afin de mesurer la force des relations modélisées, sachant que des déviations des variables construites par rapport au prix de vente pourraient survenir. Cette approche a été préférée à une approche économétrique s'enquérant davantage des liens de cause à effet entre des variables et qui aurait ipso facto nécessité un plus grand degré de confiance en une spécification particulière du modèle. L'analyse de corrélation partielle permet néanmoins d'extraire l'effet potentiel de la valeur marchande sur le prix de vente et ainsi mieux vérifier les hypothèses émises.

\section{1. Évaluation des besoins de retraite nets des cédants}

En premier lieu, suivant une approche de planification financière standard reprenant les caractéristiques de la méthode d'évaluation par revenus, une variable estimant la valeur présente des besoins de retraite nets des cédants $(\mathrm{BRN})$ a été construite. Le transfert de l'entreprise étant souvent échelonné sur plusieurs acquisitions, cette variable correspond à une fraction des besoins de retraite nets équivalente au pourcentage des parts acquises lors de l'année de la dernière acquisition de parts d'entreprise par le repreneur. L'expression suivante explicite ce premier construit :

$$
\begin{aligned}
& B R N=\omega \frac{c}{\left(\alpha_{1}-\pi\right)}+\sum_{i=1}^{I} \operatorname{proj}_{j} \frac{1}{\left(1+\alpha_{1}\right)^{t_{i}}}-y^{h f}\left[\frac{1-\left(1+\alpha_{1}\right)^{-D}}{\alpha_{1}}\right]-\text { É }- \\
& \frac{1}{\left(1+\alpha_{1}\right)^{k}} \operatorname{RRQ}\left[\frac{1-\left(1+\alpha_{1}\right)^{-x}}{\alpha_{1}}\right]-S V\left(\frac{1}{\left(1+\alpha_{1}\right)^{h}}\right)\left(\frac{1}{\alpha_{1}-\pi}\right)\left[1-\left[\frac{1+\pi}{1+\alpha_{1}}\right]^{X}\right]
\end{aligned}
$$

Où « $\omega »$ dénote le pourcentage de parts acquises, « $C »$ le coût de vie annuel du ménage du cédant, «proj » le montant requis pour le projet spécial « $i$ » (voyage, achat d'une voiture, etc.) anticipé ou réalisé « $t$ » années après la dernière transaction de parts, $\left\langle y^{h f} \gg\right.$ les revenus hors ferme annuels futurs du ménage pendant les « $\mathrm{D}$ » années séparant la dernière acquisition de parts et la retraite complète du cédant, «É $p$ » le montant d'épargne accumulé par le cédant pour sa retraite au moment de la dernière transaction de parts, « $k »$ le nombre d'années avant que le ménage du cédant touche à la rente de retraite RRQ, « $R R Q$ » le montant des prestations annuelles de la Régie des rentes du Québec,
« $X »$ l'espérance de vie du cédant ${ }^{3}$ lors de l'année de la dernière acquisition de parts, « $S V$ » le montant des prestations de retraite Sécurité Vieillesse, « $h »$ le nombre d'années avant le $65^{\mathrm{e}}$ anniversaire du cédant, « $\pi$ » le taux d'indexation des prestations Sécurité Vieillesse et « $\alpha »$ un taux d'escompte reflétant le rendement d'obligation à long terme du gouvernement canadien lors de l'année « $j »$, soit

3. L'espérance de vie choisie correspond à 19 années à l'âge de 65 ans, reflétant les statistiques nationales canadiennes. Afin de simplifier l'analyse, la même espérance de vie s'applique à chaque répondant, indépendamment de son sexe, de son revenu ou de sa santé. 
l'année précédant la dernière acquisition de parts. Le coût de vie est indexé à l'inflation $(2 \%)$ et est ramené en valeur présente sous la forme d'une perpétuité afin de tenir compte d'une éventuelle compensation envers les enfants non repreneurs. Ainsi, le cédant dispose pour chaque année des revenus d'intérêt et de dividendes de ses placements et, lors du décès, le montant excédentaire est légué en héritage, ce qui concorde avec le traitement des besoins de retraite nets perpétuels (Plamondon et Sauvé, 2002).

\section{Estimation de la valeur économique des capitaux propres de l'entreprise}

En second lieu, l'approche d'évaluation par revenus est utilisée pour construire une seconde variable dénotant la valeur économique des capitaux propres de l'entreprise (VE) ou encore la valeur présente des montants correspondant à la capacité de remboursement (CDR) moins le passif (Lepage et al., 2015) pour l'année précédant la dernière acquisition de parts d'entreprise. L'adéquation entre la fraction des parts transigées et la fraction de la valeur économique est également maintenue dans ce cas et est modélisée de la manière suivante $^{4}$ :

$$
V E=\omega\left[(C D R)\left\{\frac{1-(1+\alpha)^{-T}}{\alpha}\right\}-\text { Passif }\right]
$$

Où « $\omega »$ dénote le pourcentage de parts acquises, « $\mathrm{CDR}$ » représente la capacité de remboursement de l'entreprise (marge brute + intérêts à moyen long terme - coût

4. La capacité de remboursement utilisée est la CDR maximale, qui représente le montant maximal de l'annuité auquel peut se rapprocher une entreprise en démarrage ou en expansion. de $v i e^{5}$ ) et $« \alpha »$ un taux d'escompte reflétant le taux de rendement à long terme des obligations canadiennes. La durée de vie utile de l'entreprise «T la moyenne pondérée des durées de vie utiles des principaux actifs constituant $l^{\prime}$ 'entreprise ${ }^{6}$. Le premier terme entre crochets de l'expression représente la valeur économique de l'actif de laquelle le passif est soustrait. La variable ainsi obtenue reflète la valeur économique des capitaux propres de l'entreprise. Se basant sur l'approche d'évaluation par revenus, la valeur obtenue permet de relever, s'il y a lieu, les lacunes au plan de la gestion de l'exploitation. Contrairement à la pratique usuelle en évaluation foncière ou immobilière, la valeur de l'entreprise est contrainte à une durée fixe. Évaluer une exploitation pour laquelle les résultats bénéficiaires seraient projetés infiniment dans le futur et capitalisés selon le taux choisi se prêterait mal au contexte de négociation décrit plus haut. Une capitalisation sans durée déterminée impliquerait que le repreneur ne devienne jamais propriétaire de la ferme, ce qui serait contraire à la logique d'entreprise. Les remboursements de l'emprunt contracté pour l'acquisition consisteraient essentiellement en paiements d'intérêts et aucune provision pour le renouvellement des amortissements ne serait prise en compte. À moins que le taux de capitalisation soit considérablement plus élevé que le taux

5. La pratique française définit la capacité de remboursement comme l'excédent brut d'exploitation + produits financiers - frais financiers de court terme - prélèvements privés. Nous notons que la modélisation aurait permis un portrait plus robuste de la situation par l'utilisation de bénéfices moyens pour les quelques années précédant l'acquisition, plutôt que pour une seule année.

6. La durée de vie moyenne pondérée des actifs est d'environ 16 ans et est calculée d'après différentes statistiques concernant le secteur agricole à l'échelle provinciale : quotas (18\%) 10 ans, animaux (9\%) 5 ans, matériel (17\%) 15 ans, terres (30\%) 20 ans, bâtiments (15\%) 25 ans et autres actifs (11\%) 10 ans (CECPA, 2011). 
d'intérêt payé sur emprunt, ce qui paraît peu probable, cette pratique ne semble pas appropriée aux circonstances. De plus, dans le but d'assurer une certaine cohérence avec le premier construit décrit plus haut, le calcul de la valeur économique utilise le même taux d'escompte que celui des besoins de retraite nets. L'usage du même taux d'escompte pose donc l'hypothèse que le coût d'opportunité est le même pour le cédant et pour le repreneur. Notons que la valeur marchande utilisée dans les deux analyses de corrélation constitue l'estimation qu'en font les répondants, vraisemblablement via une combinaison de l'approche patrimoniale et de l'approche par multiples.

\section{Collecte des données et description de l'échantillon}

Afin de collecter les données de la modélisation développée et de vérifier les hypothèses de recherche, un sondage a été effectué par l'entremise de questionnaires auto-administrés. Les participants ayant été contactés sont de jeunes agriculteurs qui étaient âgés de 18 à 40 ans lors du recensement de la relève agricole par le ministère de l'Agriculture, des Pêcheries et de l'Alimentation du Québec (MAPAQ, 2011). Seuls les agriculteurs ayant repris au moins $50 \%$ des parts de l'entreprise ont été retenus afin que la population cible soit constituée de nouveaux propriétaires majoritaires en position de décideurs dans une entreprise déjà en opération. Un premier questionnaire s'adressait au repreneur et s'enquérait principalement de sa situation financière (revenus hors ferme, montage financier au transfert, etc.) et de celle de l'entreprise principale dans laquelle il ou elle détient des parts (bilan, état des résultats, etc.). Le second questionnaire s'adressait au cédant et s'intéressait à sa situation financière en prévision de la retraite (épargne, sources de revenus envisagées, etc.). Le sondage a été réalisé de janvier à avril 2016. Les questionnaires ont été envoyés à 1659 entreprises et un seul rappel a été effectué par la poste deux semaines suivant l'envoi des questionnaires. Visant un taux de réponse d'environ $30 \%$, les auteurs prévoyaient randomiser l'échantillon obtenu par stratification le rendant ainsi probabiliste. Au total, l'échantillon comprend 248 entreprises soit $15 \%$ de la population initialement visée. La longueur des questionnaires, la sensibilité des informations demandées et le fait qu'un seul rappel ait été fait alors que deux à trois rappels sont généralement prescrits pour ce genre de sondage pourraient expliquer en partie le faible taux de participation. Les caractéristiques de la population d'intérêt pour cette étude (jeunes actionnaires majoritaires et cédants) étant essentiellement inconnues, la représentativité de l'échantillon ne peut dès lors qu'être approximée. Pour ce faire, les moyennes de certaines variables clés concernant les entreprises elles-mêmes (actif, passif, revenus et dépenses) ont été comparées aux statistiques pour l'ensemble de la population agricole québécoise. Les résultats des comparaisons de moyennes n'ont pas permis de conclure avec assurance que l'échantillon soit statistiquement représentatif de la population à l'étude ni de la population agricole québécoise en général. Étant donné le faible taux de réponse, un échantillonnage ex-post par stratification n'aurait pas été envisageable, ce qui fait en sorte que les résultats obtenus ne peuvent être inférés à la population, ni à un secteur ou une région du Québec en particulier. Face à cette situation, les auteurs ont dû choisir un seuil de significativité de $10 \%$ afin de minimiser le risque de ne pas rejeter l'hypothèse nulle à tort (erreur de type 2) (Bartlett et al., 2001). Dans le cas échéant, les seuils de significativité ont été corrigés à l'aide de l'approche de Bonferroni afin de minimiser le risque de rejeter l'hypothèse nulle à tort (erreur de type 1) (Mundfrom et al., 2006). 
Tableau 1. Statistiques descriptives des échantillons utilisés pour les deux tests d'hypothèses ( $\mathrm{H} 1$ et H2)

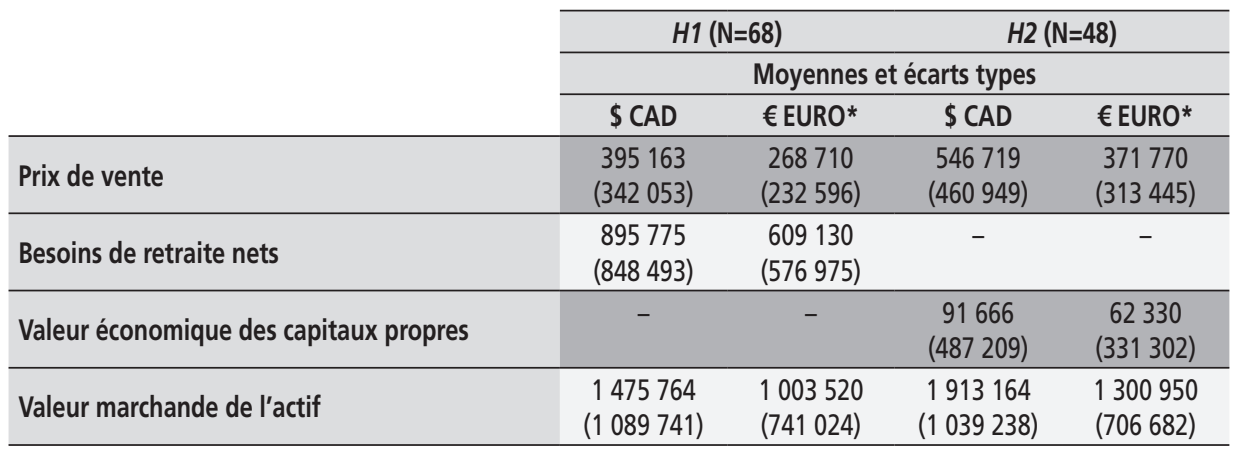

Note : * Le taux de change utilisé est de $1 \$$ canadien $\approx 0,68 €$ euro.

Source : les auteurs.

L'échantillon est principalement constitué d'entreprises œuvrant en production laitière $(55,3 \%)$, acéricole $(10,7 \%)$, céréalière, fourragère et protéagineuse $(8,8 \%)$, en élevage de bovins de boucherie $(7,9 \%)$, de porcs $(4,7 \%)$, en production de légumes et fruits $(3,7 \%)$ et en élevage de volaille $(2,8 \%)$. La surface de terres cultivables détenues dans l'ensemble de l'échantillon est d'en moyenne 91 hectares. L'exploitation laitière moyenne de l'échantillon possède un droit de production de $37,1 \mathrm{~kg}$ de matière grasse par jour, d'une valeur d'environ $640000 €$. L'âge moyen des repreneurs lors de leurs dernières acquisitions de parts d'entreprise était de 28,55 ans et de 57,31 ans pour les cédants.

Les observations manquantes et le retrait des données aberrantes ont réduit la taille de l'échantillon utilisé pour tester les deux hypothèses de recherche $(\mathrm{N}=68$ pour $\mathrm{H} 1$ et $\mathrm{N}=48$ pour $H 2$ ). Le tableau 1 présente une description des principales données des deux tests effectués. Le prix de vente moyen dans le sous-groupe de l'échantillon utilisé pour cette première partie de l'analyse est de $268710 €$, la part des besoins de retraite nets des cédants correspondant au pourcentage des parts acquises est en moyenne de $609130 €$ et la valeur marchande moyenne de l'actif est de $1003520 €$. Dans le cas de la deuxième partie de l'analyse, le prix de vente moyen est de $371770 €$ dans ce sous-groupe de l'échantillon, la part de la valeur économique des capitaux propres de l'entreprise correspondant au pourcentage des parts acquises est en moyenne de $62330 €$ et la valeur marchande moyenne est de $1300950 €^{7}$.

Le tableau 2 décrit l'estimation des auteurs quant au rapport entre valeurs économiques et valeurs marchandes dans l'échantillon pour l'année 2014. Du côté de l'actif, le ratio prend une valeur moyenne relativement faible et nous constatons que le secteur laitier $(47,7 \%)$ ne semble pas différer substantiellement des autres types de production $(49,4 \%)$. Cependant, le ratio pour les capitaux propres montre une situation considérablement différente avec le secteur laitier affichant un ratio relativement plus élevé (12,5\%) que pour les autres types de production $(6,6 \%)$. Ces estimations semblent conséquentes avec la tendance décrite par Perrier (2013) dans le cas des fermes laitières qui en 2011

7. La valeur marchande moyenne dans ce sousgroupe est sensiblement plus élevée que dans le sous-groupe précédent, principalement à cause de la présence d'une ferme fortement capitalisée, absente de l'analyse précédente. 
RECHERCHE

David OUELLET, Jean-Philippe PERRIER

Tableau 2. Valeurs économiques en pourcentage des valeurs marchandes

\begin{tabular}{lcccc}
\hline & $\begin{array}{c}\text { Production } \\
\text { laitière }\end{array}$ & $\begin{array}{c}\text { Autres } \\
\text { productions }\end{array}$ & Total & $\begin{array}{c}\text { Perrier } \\
(2013)\end{array}$ \\
\hline Valeur économique de l'actif sur valeur & $47,7 \%$ & $49,4 \%$ & $48,5 \%$ & $55 \%$ \\
marchande de l'actif & $\mathrm{N}=32$ & $\mathrm{~N}=29$ & $\mathrm{~N}=61$ & $\mathrm{~N}=74$ \\
\hline Valeur économique des capitaux propres & $12,5 \%$ & $6,6 \%$ & $9,9 \%$ & $20 \%$ \\
sur valeur marchande des capitaux propres & $\mathrm{N}=31$ & $\mathrm{~N}=25$ & $\mathrm{~N}=56$ & $\mathrm{~N}=74$ \\
\hline
\end{tabular}

Source : les auteurs.

affichaient un ratio d'environ $55 \%$ pour la valeur économique de l'actif sur sa valeur marchande et d'environ $20 \%$ pour les capitaux propres.

\section{Résultats et discussion}

La première partie de l'analyse vérifie l'hypothèse voulant que le prix de vente soit corrélé avec les besoins de retraite nets des cédants, indépendamment de la valeur marchande. Les résultats de l'analyse de corrélation partielle figurent au tableau 3 et montrent un coefficient de 0,402 statistiquement significatif ( $p=0,001<0,1$ ). Des coefficients de corrélations bivariées entre les trois variables sont également présentés. En utilisant l'approche de correction de Bonferroni ${ }^{8}$, des valeurs $p$ de moins de 0,033 étaient nécessaires afin d'obtenir la significativité statistique pour ces corrélations. La valeur marchande est significativement corrélée avec le prix de vente $(\mathrm{r}(66)=0,315 ; p=0,009)$ et avec le prix reflétant les besoins de retraite nets $(\mathrm{r}(66)=0,334 ; \quad p=0,005) . \quad$ Cependant, une fois l'effet de ces corrélations extrait, la corrélation entre le prix de vente et le prix reflétant les besoins de retraite nets $(\mathrm{r}(66)=0,465 ; p=0,000)$ demeure statistiquement significative, à un seuil de $1 \%$.

Les résultats obtenus montrent que le prix de vente est effectivement corrélé avec les besoins de retraite nets des cédants, et ce même sans l'effet de cause commune

8. Le seuil de significativité a été divisé par le nombre de corrélations bivariées testées soit trois. provenant de la valeur marchande de l'entreprise. L'hypothèse nulle d'absence de corrélation en $H 1$ est donc rejetée. Il est cependant à noter qu'une fois cet effet extrait, la force du lien entre le prix de vente et les besoins de retraite nets est plus faible, passant d'un coefficient de 0,465 à 0,402 . Par ailleurs, le prix auquel sont vendues les parts d'entreprise ne semble pas suffire aux besoins de retraite des cédants puisqu'il couvre en moyenne $63 \%$ (erreur standard de 8,2\%) des besoins de retraite nets. De plus, la moyenne de ce ratio n'est pas significativement différente $(\mathrm{F}(1 ; 40)=1,132 ; p=0,294)$ dans le secteur laitier $(68,8 \%)$ par rapport aux autres types de production $(50,2 \%)^{9}$. Le manque à gagner en termes de financement de la retraite serait donc relativement similaire pour l'ensemble des cédants de l'échantillon. En outre, la valeur marchande représente en moyenne $103 \%$ des besoins de retraite nets (erreur standard de 9,04\%). Ce ratio est significativement plus élevé $(\mathrm{F}(1 ; 27)=3,638 ; \quad p=0,067)$ pour les fermes laitières $(107 \%)$ que pour les autres types de production (67\%), étant donné la plus grande capitalisation des fermes laitières. Si la ferme est synonyme de « fonds de retraite » pour les agriculteurs cédants, il semble que ce soit davantage le cas pour les producteurs laitiers. Cependant, afin de favoriser la pérennité de l'entreprise, le cédant récupérera en moyenne une fraction

9. Tous les tests de comparaisons de moyennes rapportés dans cet article ont été effectués avec l'approche Brown-Forsythe. 
Tableau 3. Corrélation partielle : prix de vente, besoins de retraite nets et valeur marchande de l'actif $(\mathrm{N}=68)$

\begin{tabular}{|c|c|c|c|c|c|}
\hline $\begin{array}{l}\text { Variables } \\
\text { de contrôle }\end{array}$ & & & $\begin{array}{c}\text { Prix } \\
\text { de vente }\end{array}$ & $\begin{array}{l}\text { Besoins de retraite } \\
\text { nets }\end{array}$ & $\begin{array}{c}\text { Valeur marchande } \\
\text { de l'actif }\end{array}$ \\
\hline \multirow[t]{9}{*}{-aucune- } & \multirow[t]{3}{*}{ Prix de vente } & Corrélation & 1,000 & $* * *, 465$ & $* *, 315$ \\
\hline & & Sig. (bilat.) & &, 000 &, 009 \\
\hline & & Ddl & 0 & 66 & 66 \\
\hline & \multirow{3}{*}{$\begin{array}{l}\text { Besoins de retraite } \\
\text { nets }\end{array}$} & Corrélation & & 1,000 & **,334 \\
\hline & & Sig. (bilat.) & & &, 005 \\
\hline & & Ddl & & 0 & 66 \\
\hline & \multirow{3}{*}{$\begin{array}{l}\text { Valeur marchande } \\
\text { de l'actif }\end{array}$} & Corrélation & & & 1,000 \\
\hline & & Sig. (bilat.) & & & \\
\hline & & Ddl & & & 0 \\
\hline \multirow{6}{*}{$\begin{array}{l}\text { Valeur marchande } \\
\text { de l'actif }\end{array}$} & \multirow[t]{3}{*}{ Prix de vente } & Corrélation & 1,000 & $* * *, 402$ & \\
\hline & & Sig. (bilat.) & &, 001 & \\
\hline & & Ddl & 0 & 65 & \\
\hline & \multirow{3}{*}{$\begin{array}{l}\text { Besoins de } \\
\text { retraite nets }\end{array}$} & Corrélation & & 1,000 & \\
\hline & & Sig. (bilat.) & & & \\
\hline & & Ddl & & 0 & \\
\hline
\end{tabular}

Notes : *** significatif à $\alpha=1 \%$; ** significatif à $\alpha=5 \%$; * significatif à $\alpha=10 \%$ (avec correction Bonferroni).

Source : les auteurs.

de la valeur marchande de la ferme et ce, même si cela signifie un niveau de vie plus modeste à la retraite. Nous pouvons donc en déduire que le prix soit effectivement limité par la capacité de remboursement.

La deuxième partie de l'analyse vérifie l'hypothèse voulant que le prix de vente soit corrélé avec la valeur économique des capitaux propres de l'entreprise, indépendamment de la valeur marchande. Les résultats de l'analyse de corrélation sont présentés au tableau 4. La corrélation bivariée entre le prix de vente et la valeur économique des capitaux propres est statistiquement significative $(\mathrm{r}(46)=0,340$; $p=0,018)$, mais une fois l'effet des autres corrélations extrait, la corrélation partielle ne tient plus de manière statistiquement significative puisque $p=0,132>0,10$. La corrélation entre la valeur marchande et le prix de vente $(\mathrm{r}(46)=0,493 ; p=0,000)$ ainsi que celle avec la valeur économique des capitaux propres $(\mathrm{r}(46)=0,316$; $p=0,029)$ semblent donc dominer la relation entre prix de vente et valeur économique.

Les résultats obtenus montrent que le prix de vente est corrélé avec la valeur économique des capitaux propres de l'entreprise, mais de façon moins significative que dans le cas des besoins de retraite nets. De plus, le lien entre prix et valeur économique devient considérablement plus faible $(0,223$ par rapport à 0,340$)$ en contrôlant la valeur marchande de l'entreprise. La dispersion de la variable dénotant la valeur économique est relativement grande (écart type de $331302 €$ ), ce qui pourrait expliquer le manque de significativité de sa relation avec le prix de vente. L'hypothèse nulle de non-corrélation en $H 2$ entre le prix de vente et la valeur économique des capitaux propres de l'entreprise ne peut donc être rejetée. Ce résultat doit toutefois être interprété dans le contexte des résultats de la première partie de l'analyse. Afin d'assurer le transfert intergénérationnel, les cédants ne peuvent demander un prix qui 
RECHERCHE

David OUELLET, Jean-Philippe PERRIER

Tableau 4. Corrélation partielle ; prix de vente, valeur économique des capitaux propres et valeur marchande de l'actif ( $\mathrm{N}=48)$

\begin{tabular}{|c|c|c|c|c|c|}
\hline $\begin{array}{l}\text { Variables } \\
\text { de contrôle }\end{array}$ & & & $\begin{array}{l}\text { Prix de } \\
\text { vente }\end{array}$ & $\begin{array}{l}\text { Valeur écono- } \\
\text { mique des capi- } \\
\text { taux propres }\end{array}$ & $\begin{array}{l}\text { Valeur marchande } \\
\text { de l'actif }\end{array}$ \\
\hline \multirow[t]{9}{*}{-aucune- } & \multirow[t]{3}{*}{ Prix de vente } & Corrélation & 1,000 & $*, 340$ & $* * *, 493$ \\
\hline & & Sig. (bilat.) & &, 018 &, 000 \\
\hline & & Ddl & 0 & 46 & 46 \\
\hline & \multirow{3}{*}{$\begin{array}{l}\text { Valeur } \\
\text { économique des } \\
\text { capitaux propres }\end{array}$} & Corrélation & & 1,000 & *,316 \\
\hline & & Sig. (bilat.) & & &, 029 \\
\hline & & Ddl & & 0 & 46 \\
\hline & \multirow{3}{*}{$\begin{array}{l}\text { Valeur marchande } \\
\text { de l'actif }\end{array}$} & Corrélation & & & 1,000 \\
\hline & & Sig. (bilat.) & & & \\
\hline & & Ddl & & & 0 \\
\hline \multirow{6}{*}{$\begin{array}{l}\text { Valeur marchande } \\
\text { de l'actif }\end{array}$} & \multirow[t]{3}{*}{ Prix de vente } & Corrélation & 1,000 & ,223 & \\
\hline & & Sig. (bilat.) & & , 132 & \\
\hline & & Ddl & 0 & 45 & \\
\hline & \multirow{3}{*}{$\begin{array}{l}\text { Valeur } \\
\text { économique des } \\
\text { capitaux propres }\end{array}$} & Corrélation & & 1,000 & \\
\hline & & Sig. (bilat.) & & & \\
\hline & & Ddl & & 0 & \\
\hline
\end{tabular}

Notes : *** significatif à $\alpha=1 \%$; ** significatif à $\alpha=5 \%$; * significatif à $\alpha=10 \%$ (avec correction Bonferroni).

leur permettrait de combler entièrement leurs besoins de retraite. Le fait que le prix soit inférieur à la valeur marchande et inférieur aux besoins de retraite nets indique que la capacité de payer de l'entreprise est limitée. En effet, le prix de vente semble être le maximum que puisse supporter l'entreprise puisque le ratio prix de vente sur valeur économique est en moyenne de $115,6 \%$, bien qu'il varie considérablement (erreur standard de 71,93\%). Par ailleurs, nous ne pouvons conclure que la moyenne de ce ratio soit significativement différente $(\mathrm{F}(1 ; 22)=0,410 ; \quad p=0,529)$ dans le secteur laitier par rapport aux autres types de production. Si le prix est en moyenne supérieur à la valeur économique des capitaux propres, cela pourrait signifier que l'emprunt comme composante du montage financier au transfert correspond à seulement une partie du prix de vente total. Ce résultat montre néanmoins que des compromis considérables sont réalisés des deux côtés de la transaction en vue de favoriser la continuité de l'entreprise, puisqu'en moyenne les produits de la vente ne comblent qu'une partie des besoins de retraite nets des cédants et que les repreneurs paient plus que la valeur économique des capitaux propres de l'entreprise.

Ces résultats pourraient expliquer les constats décrits par Perrier et al. (2015) qui mentionnent que dans approximativement $90 \%$ des cas de transferts, le recours à des services conseils afin d'évaluer les besoins de retraite des cédants et la capacité de remboursement des entreprises sont utilisés afin de déterminer le prix de vente (Perrier et al., 2015). Le degré de complexité du calcul et l'absence de marge de manœuvre pourraient agir comme incitatifs à utiliser ces services et ainsi éviter les conflits qui pourraient émerger entre cédants et repreneurs en cas de désaccord sur le prix de vente. Cependant, en supposant que plusieurs conflits émergent des contraintes financières et de l'absence d'une marge de manœuvre suffisante qui eux découlent en partie des décisions de gestion financière et d'investissement 
passées et non pas de la détermination même du prix de vente, la perspective de discordes futures demeure. Considérer l'évaluation de l'exploitation agricole dans le contexte particulier de la transmission (Barthélémy, 1997 ; Debruyne, 2010) pourrait dès lors permettre d'identifier les obstacles potentiels et prévenir les insuccès. Par ailleurs, l'absence de différences significatives entre les fermes laitières et les fermes provenant des autres secteurs suggère que la problématique du prix de vente semble davantage une question de gestion plutôt qu'une question spécifique au secteur. En ce sens, les conflits ou les compromis découlant de la détermination du prix de vente pourraient être plus probables pour les entreprises dont l'écart entre valeurs économique et marchande est le plus prononcé. La non-représentativité de notre échantillon ne permet toutefois pas de conclure avec certitude de la vraisemblance d'un tel constat. Plus de recherche quant au lien entre de bonnes pratiques de gestion financière en amont du processus de transfert et l'aisance avec laquelle cédants et repreneurs s'accordent dans la prise de décision apparaît nécessaire.

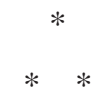

L'importance du don dans le montage financier au transfert d'entreprises agricoles québécoises est prépondérante et risque de l'être encore plus à l'avenir. La baisse relative de la valeur économique des fermes montre un problème structurel de faible rentabilité aggravé par un endettement inopportun et masqué par des valeurs marchandes en constante augmentation. Si la réelle valeur des exploitations agricoles s'exprime lors de leur transmission, il semblerait que les agriculteurs eux-mêmes ne soient pas en mesure d'en rendre compte. Les résultats de la présente étude montrent effectivement que, comme les produits de la vente de la ferme ne comblent en moyenne qu'une partie des besoins de retraite nets, et que le montant payé par le repreneur semble correspondre au montant maximum que puisse supporter l'entreprise, les compromis présentement réalisés ne peuvent assurer la pérennité de l'entreprise et risquent d'être encore plus contraignants lors des prochains transferts intergénérationnels. Notre échantillon n'est cependant pas représentatif sur le plan statistique et les résultats de cette étude ne peuvent donc pas être généralisés à l'ensemble de la population visée. De plus, l'approche développée dans cette étude n'élimine pas entièrement les écueils de l'évaluation d'entreprise et de la planification financière. La prise en compte d'hypothèses plus restrictives et l'utilisation de données plus précises pourraient permettre des résultats plus robustes. Chose certaine, si la tendance se maintient quant à l'écart entre les valeurs marchande et économique des entreprises, nous pourrions continuer de constater une réduction du nombre de fermes et une dévitalisation des communautés rurales du Québec. 


\section{RÉFÉRENCES BIBLIOGRAPHIQUES}

Barclay E., Foskey R., Reeve I. J. (2007). Farm Succession And Inheritance: Comparing Australian And International Trends. (1741514630). Rural Industries Research And Development Corporation: Commonwealth Of Australia.

Barthélémy D. (1997). Évaluer l'entreprise agricole. Paris, PUF.

Bartlett J., Kotrlik J., Higgins C. (2001). Organizational Research: Determining Appropriate Sample Size in Survey Research Appropriate Sample Size in Survey Research. Information Technology, Learning, And Performance Journal, vol. $19, \mathrm{n}^{\circ} 1$, pp. 43.

Cantin S., Lepage F. (2006). Le montage financier d'un transfert de ferme et son lien avec la pérennité de l'entreprise : revue de littérature. Université Laval, Traget Laval.

Cecpa (2011). Étude sur les coûts de production, céréales, maïs-grain et oléagineux en 2009 au Québec. Lévis, Qc Canada, Centre d'études sur les coûts de production en agriculture.

Damodaran A. (2001). The Dark Side of Valuation: Valuing Old Tech, New Tech And New Economy Companies. Harlow, Financial Times Prentice Hall.

Debruyne M. (2010). Valeur, performance et efficacité productive de l'entreprise agricole. La Revue des sciences de gestion, direction et gestion, vol. 45, $\mathrm{n}^{\circ}$ 243-244, pp. 89-102.

Eves C. (2007). Current Rural Valuation Practice: A Survey of Valuers And Agribusiness Managers On Farm Management And Sustainable Rural Land Use. Papier présenté à Sustainable Human Settlements For Economic And Social Development, Livingston, Zambia.

Feldman S. J. (2005). Principles of Private Firm Valuation. Hoboken, NJ, John Wiley $\&$ Sons.

Gaté R., Latruffe L. (2016). Difficultés rencontrées lors de la transmission d'exploitations agricoles. Le cas de la Bretagne. Économie rurale, $\mathrm{n}^{\circ} 351$, pp. 5-24.
Handfield M. (2006). Étude des facteurs culturels et sociaux dans l'abandon du processus de succession au sein des entreprises agricoles familiales : analyse des logiques et des stratégies partenaires à partir de la perspective des prédécesseurs familiaux. Doctorat, Université du Québec à Rimouski, 922 p.

Handfield M., Boisjoly J., Jean B. (2008). Les logiques et stratégies d'équité et de financement du transfert de la ferme familiale. Ministère de l'Agriculture, des Pêcheries et de l'Alimentation du Québec.

Hargreaves B., Mccarthy I. (2010). Is New Zealand Farm Land Worth What It Will Produce? Papier présenté à Pacific Rim Real Estate Society Conference 2010, Wellington, Nz.

Jacques-Jouvenot D., Schepens F. (2007). Transmettre et reprendre une entreprise: de l'Homo EEconomicus à l'Homo Memor. Revue du Mauss, $\mathrm{n}^{\circ}$ 1, pp. 377-391.

Lepage F., Couderc J.-P., Perrier J.-P., Parent D. (2011). Transfert: les déterminants de la performance des exploitations agricoles familiales. Économie rurale, n ${ }^{\circ} 324$, pp. 3-17.

Lepage F., Perrier J.-P., Montel B. (2015). La capacité financière des exploitations agricoles à être transférées : quels horizons pour les fermes laitières québécoises? Revue canadienne des sciences régionales, $\mathrm{n}^{\circ} 38$, $52 \mathrm{p}$.

Lobley M. (2010). Succession in The Family Farm Business. Journal of Farm Management, vol. 13, $\mathrm{n}^{\circ} 12$, pp. 839-851.

Ma S., Swinton S. M. (2012). Hedonic Valuation of Farmland Using Sale Prices Versus Appraised Values. Land Economics, vol. $88, \mathrm{n}^{\circ} 1$, pp. 1-15.

Mapaq (2011). Portrait de la relève agricole au Québec. Québec, Ministère de l'Agriculture, des Pêcherires et de l'Alimentation du Québec.

Meloche J.-P., Debailleul G. (2013). Acquisition des terres agricoles par des non-agriculteurs au Québec: ampleur, causes et portée du phénomène. Montréal, Qc Canada, Cirano. 
Mishkin F. S. (2008). Comment réagir face aux bulles des prix d'actifs? Revue de la stabilité financière, $\mathrm{n}^{\circ} 12$.

Montel B., Sanscartier R. (2013). Proposition d'outils financiers ou d'alternatives favorisant le transfert de fermes et répondant aux besoins des producteurs agricoles. Écoressources Inc.

Mundfrom D. J., $\quad$ Perrett J. J., Schaffer J., Piccone A., Roozeboom M. (2006). Bonferroni Adjustments In Tests For Regression Coefficients. Multiple Linear Regression Viewpoints, vol. 32, n 1, pp. 1-6.

Murray W. G. (1969). Farm Appraisal And Valuation. Ames, Iowa State University Press, 5th Ed.

Parent D., Jean B., Simard M. (2000). Transmission et maintien de la ferme familiale: analyse des facteurs et stratégies d'établissement de la relève agricole familiale via les situations d'insuccès. Sainte-Foy, Qué : Pavillon Paul-Comtois, Université Laval.

Perrier J.-P., Allard I., Parent D. (dir.). (2004). Transfert de ferme et démantèlement au
Québec : études de cas. Québec, Qc Canada, Université Laval, Traget Laval.

Perrier J.-P., Lepage F., Ouellet D. (2015). Fixation du prix de vente au transfert des exploitations agricoles québécoises: le point de vue des conseillers et des agriculteurs. Québec, Qc Canada: Université Laval, Traget Laval.

Perrier J.-P. (2013). Comment s'établir autrement. Colloque «Établissement et retrait de l'agriculture, drummondville », QC.

Pitts M., Fowler C., Kaplan M., Nussbaum J., Becker J. (2009). Dialectical Tensions Underpinning Family Farm Succession Planning. Journal of Applied Communication Research, vol. 37, n 1 , pp. 59-79.

Plamondon R. G., Sauvé P. (2002). La planification financière personnelle : une approche globale et intégrée. Boucherville, Gaëtan Morin, 4 éd.

Tondreau J., Parent D., Perrier J.-P. (2002). Transmettre la ferme familiale d'une génération à l'autre: situation au Québec et regard sur le monde. Québec, Université Laval, Agri-Gestion Laval. 